\title{
The ER/Golgi interface - is there anything in-between?
}

\author{
Chris Hawes* \\ Plant Cell Biology, Department of Biological and Medical Sciences, Oxford Brookes University, Oxford, UK \\ *Correspondence: chawes@brookes.ac.uk
}

\section{INTRODUCTION}

Endoplasmic reticulum export sites (ERES) are specific domains on the ER surface where cargo proteins, both soluble and membrane-bound, exit the ER in transit to the cis-side of the Golgi apparatus for processing and secretion. In mammalian cells, ERES can be morphologically recognized as areas on the rough ER membrane that are devoid of ribosomes and form coated buds, vesicles, and vesicular tubular clusters (Tang et al., 2005). In most instances, the clusters undergo long-range transport mediated by microtubules to dock with the cis-face of the Golgi (Tang et al., 2005). In terms of their protein complement the ERES is best characterized by the components of the COPIIcoated membranes, the $\mathrm{v}$-SNARES required for docking to the target membranes and p24 proteins, putative cargo receptors (Strating and Martens, 2009). In yeast, it is also thought that COPII-coated vesicles bud from the ER membrane to transport cargo to very simple Golgi bodies that mostly exist as single cisternae and have the capacity to mature from cis- through to trans-forms (Matsuura-Tokita et al., 2006).

The situation in plants however is considerably more confusing. Over the years, conventional transmission electron microscopy (TEM) of higher plants has not revealed the presence of visible coated areas on ER membranes or any structures that could be convincingly interpreted as ERES. Over the past decade however, the application of ultra-rapid freezing techniques combined with tomographic electron microscopy has suggested that a COPII vesicle mediated transport may indeed exist (Kang and Staehelin, 2008). At the same time a live cell imaging approach based around the expression of fluorescent protein markers indicated that Golgi bodies are intimately associated with the ER, and that the two organelles may even be connected together (Boevink et al., 1998; daSilva et al., 2004; Faso et al., 2009). These observations developed into the "secretory unit" concept of Golgi and ER exit sites moving in tandem with the ER (daSilva et al., 2004) and that Golgi biogenesis could be from newly generated exit sites (Hawes et al., 2008). Immunolabeling of COPII components has suggested that exit site proteins could exist on the ER separate from Golgi bodies (Yang et al., 2005; Zhang et al., 2010), although almost all data from live cell imaging suggests a close association between the two structures (Hanton et al., 2009; Langhans et al., 2012). Such differences could be due to fixation during immunolabeling procedures or expression levels of constructs in live cell imaging.

In most plant cells, the ER exists as two interchangeable forms, tubular and cisternal, and in differentiated cells the former tends to predominate. Interestingly Golgi bodies are associated with ER tubules and the curved rims of cisternae, but not with the cisternal surface (Sparkes et al., 2009a). Thus, it appears that in plants ERES are restricted to ER membranes which exhibit a high degree of curvature. Such curvature has been shown to be, at least in part, regulated by the membrane curvature inducing reticulon family of proteins which reside in a "W" topology in the ER membrane (Sparkes et al., 2010; Tolley et al., 2010). Interestingly another ER-associated protein, the mammalian atlastin homolog RHD3, has been reported not only to be involved in maintaining the organization of the tubular ER network, but also in Golgi distribution and movement (Chen et al., 2011; Stefano et al., 2011). Expression of dominant negative mutants of the GTPase apparently resulted in a slowing of Golgi movement and in Golgi clustering (Chen et al., 2011). However, it has yet to be shown if either of these groups of proteins is involved in tethering Golgi bodies to the curved ER membrane surface.

Recently a small family of two ER membrane proteins, again with multiple membrane spanning domains, has been implicated in the function of ERES. Overexpression of Arabidopsis thaliana KMS1 and KMS2 and mutant forms had a major effect on Golgi organization, movement, and secretion (Wang et al., 2011). Like the reticulons and RHD3, it is not yet known if these proteins directly interact with any ERES or Golgi components.

\section{EVIDENCE FOR VESICLE MEDIATED TRANSPORT BETWEEN PLANT ER AND GOLGI - ANTEROGRADE TRANSPORT}

The evidence for COPII vesicle mediated transport in plants is not overwhelming, even though all the molecular components for the COPII coat have been identified by bioinformatics analyses (Robinson et al., 2007). Certainly, coating of a membrane does not necessarily indicate the presence of free vesicles. Recently elegant in vitro experiments from the Schekman lab. have shown that when giant unilamellar membrane vesicles are incubated with COPII components tabulation of the membrane results with a bead-like appearance, not in the formation of vesicles (Bacia et al., 2011). The number of reports of COPII buds on the ER surface and of free COPII vesicles in plant tissues is limited (Langhans et al., 2012) and restricted to material such as meristematic cells and Arabidopsis endosperm that has been stabilized for TEM by high pressure freezing, and often analyzed using electron tomography (Kang and Staehelin, 2008; Hwang and Robinson, 2009). The question that has to be asked is why conventional EM fixation preserves clathrin, COPI coats, and even ERES in mammalian cells but not exit sites or COPII coats on vesicles in plant tissues?

\section{EVIDENCE FOR VESICLE MEDIATED TRANSPORT BETWEEN PLANT ER AND GOLGI - RETROGRADE TRANSPORT}

It has long been known that COPI vesicles are associated with plant Golgi bodies (Paul and Frigerio, 2007) and it is assumed that as in mammalian cells they are most likely involved in retrograde transport. Their function is also required to maintain the integrity of the ERES (Stefano et al., 2006). However, progress in this field has been 
severely hampered by the lack of any reliable retrograde markers available to plant cell biologists. It has been assumed that as the cis-Golgi-ER interface is so close, retrograde transport takes place at the ERES, but we have no structural or functional evidence for this. It is well established that in many plant tissues, with the possible exception of Arabidopsis roots which appear to have a BFA resistant ARF1 guanine-nucleotide exchange factor GNL1 (Richter et al., 2007), that blockage of secretion with Brefeldin A (BFA) results in the redistribution of Golgi membrane into the ER followed by the dissolution of individual Golgi stacks (Saint-Jore et al., 2002; Robinson et al., 2008). Such an effect can also be seen with blockage of secretion by expression of a GTP-locked version of the Sar1p-GTPase which is responsible for the initiation of COPII coat assembly (daSilva et al., 2004; Osterrieder et al., 2010). What was surprising however, was the recent report that the result of such a secretory blockage results in a retrograde absorption of Golgi enzymes into the ER starting at the trans-face, with cis-located markers being the last to disappear (Schoberer et al., 2010). The implication being, that there is either a direct route from the trans-Golgi to the ER (defined import sites?) or that a retrograde pathway transporting membrane-bound proteins through the Golgi stack exists. This opens up the intriguing possibilities that transport back to the ER could be via tubules missing the export sites, by a sequential production of retrograde COPI vesicles or even by a direct flow of membrane proteins back through the stack. On reformation of Golgi stacks, cis-Golgi marker enzymes appear first. Interestingly, electron microscopy of Golgi body reformation has shown that structurally the clusters of membranes that appear first on BFA washout are morphologically more similar to the trans-Golgi face with associated clathrin vesicles, than to cis-cisternae (Langhans et al., 2007). These pathways still remain to be explored in detail.

\section{EVIDENCE FOR A DIRECT LINKAGE BETWEEN PLANT ER AND GOLGI}

The advent of fluorescent protein technology has undoubtedly revolutionized our concept of the organization and dynamics of plant cell cytoplasm. Utilizing this we have shown that in leaves not only does the
ERES/Golgi complex move along the ER, it can move in the same direction as the ER membrane and the movement is somehow myosin mediated (Runions et al., 2006; Sparkes et al., 2009a). This has led us to postulate that there must be a direct linkage between the Golgi and the ER. In support of this, we have recently demonstrated that it is possible to capture and manipulate individual Golgi bodies in Arabidopsis leaf epidermal cells using a focused infra-red laser beam (so-called laser tweezers). In epidermal cells expressing red fluorescent protein tagged Golgi bodies and green fluorescent protein tagged ER, Sparkes et al. (2009b) demonstrated that when individual Golgi bodies were pulled around the cortical cytoplasm, they dragged behind them tubules of ER. Thus, many microns of apparently new ER tubules could be weaved around the cytoplasm. The key conclusion to be drawn from this study was that Golgi bodies are indeed physically attached to the ER. Interestingly, on the rare occasion that a Golgi body pulled free from the ER, it could be reconnected to the end of a free ER tubule and that tubule could be extended by lateral movement of the trap. This indicates the potential presence of tethering proteins on the ER or Golgi surfaces, and several reports have highlighted the existence of plant homologs of well characterized cis-Golgi matrix or tethering proteins such as CASP, golgin-84, and p115 (Renna et al., 2005; Latijnhouwers et al., 2007; Kang and Staehelin, 2008; Schoberer et al., 2010). However, even with such tethering we still need to solve the matter of membrane continuity between the ER and Golgi. Is there enough physical space between the ER membrane and a cis-cisterna for a vesicle to undergo scission from its donor membrane and docking onto its acceptor? According to Langhans et al. (2012) this is indeed the case. Conversely, are there transient but direct membrane connections between the two organelles with COPII components initiating the site and process tabulation (Bacia et al., 2011) and concentrating cargo at the exit site? Recently a careful analysis of the ER-Golgi interface in tobacco leaf epidermal cells concluded that confocal microscopy reveals an accumulation of COPII components (suggested to be vesicles) adjacent to the cis-Golgi face rather than on the ER surface (Langhans et al., 2012). When a Golgi body is observed in side view there appears to be a small gap between the ER and the cis-face where fluorescence of XFP-tagged COPII components is observed. Calculations show that such a fluorescence signal could be generated by tagged proteins on a small number of COPII vesicles. Therefore, the chances of observing one or more of these vesicles in a 70-nm resin section for EM are very low. Whilst this may be true, such data does not in itself prove the existence of COPII vesicles at the cis-face and the argument ignores the fact that such vesicles should be observed in EM section cut parallel to the surface of cis-cisternae rather than the classic micrographs longitudinal sections through the Golgi stack. This then poses the question on why such COPII components cannot be imaged on the ER surface? Are they simply at too low a concentration to produce a signal or does the ER membrane bud before coating?

Of course it is also possible that different cell types may exhibit different ER-Golgi relationships. For instance most fluorescent protein studies of ER-Golgi interactions have been in highly vacuolated cell types whereas reports of COPII vesicles have come from more densely cytoplasmic cells, such as those in meristems, which show less Golgi movement and are more difficult to study by live cell imaging (Kang and Staehelin, 2008).

\section{FUTURE PROSPECTS}

To answer the problems posed in this short article it is clear that we not only need to identify the interactome of all the proteins at the ER/Golgi interface, we need much greater resolution in our confocal microscopy than can be rendered by standard techniques. We are entering an exciting era where a whole host of high resolution lightbased imaging technologies are becoming generally available including advances in optical technology some of which break the diffraction barrier of light, such as STED, structured illumination, and light sheet fluorescence microscopy (Maizel et al., 2011), through to the PALM and STORM techniques based around the photoactivation of individual fluorescent molecules. Hopefully, one or more of these will soon be adaptable to plant tissue to offer nanometer scale resolution to differentiate the distribution of fluorescent probes at the ER-Golgi interface. Likewise new electron microscopies are coming on line such as ultra-high 
resolution cryo-SEM and focused ion beam etching combined with high resolution SEM which may permit a more detailed picture of ER-Golgi interactions in different cell types both in 3-D and at high resolution.

\section{REFERENCES}

Bacia, K., Futai, E., Prinz, S., Meister, A., Daum, S., Glatte, D., Briggs, J. A. G., and Schekman, R. (2011). Multibudded tubules formed by COPII on artificial liposomes. Sci. Rep. 1. doi: 10.1038/srep00017

Boevink, P., Oparka, K., Santa Cruz, S., Martin, B., Betteridge, A., and Hawes, C. (1998). Stacks on tracks: the plant Golgi apparatus traffics on an actin/ER network. Plant J. 15, 441-447.

Chen, J., Stefano, G., Brandizzi, F., and Zheng, H. (2011). Arabidopsis RHD3 mediates the generation of the tubular ER network and is required for Golgi distribution and motility in plant cells. J. Cell. Sci. 124, 2241-2252.

daSilva, L. L. P., Snapp, E. L., Denecke, J., LippincottSchwartz, J., Hawes, C., and Brandizzi, F. (2004). Endoplasmic reticulum export sites and Golgi bodies behave as single mobile secretory units in plant cells. Plant Cell 16, 1753-1771.

Faso, C., Chen, Y.-N., Tamura, K., Held, M., Zemelis, S., Saravanan, R., Marti, L., Hummel, E., Kung, L., Miller, E., Hawes, C., and Brandizzi, F. (2009). A missense mutation in the Arabidopsis COPII coat protein Sec24A induces the formation of clusters of the endoplasmic reticulum and Golgi apparatus. Plant Cell 21, 3655-3671.

Hanton, S. L., Matheson, L. A., Chatre, L., and Brandizzi, F. (2009). Dynamic organization of COPII coat proteins at endoplasmic reticulum export sites in plant cells. Plant J. 57, 963-974.

Hawes, C., Osterrieder, A., Hummel, E., and Sparkes, I. (2008). The plant ER-Golgi interface. Traffic 9, 1571-1158.

Hwang, I., and Robinson, D. G. (2009). Transport vesicle formation in plant cells. Curr. Opin. Plant Biol. $12,1-10$.

Kang, B. H., and Staehelin, L. A. (2008). ER-to-Golgi transport by COPII vesicles in Arabidopsis involves a ribosome excluding scaffold that is transferred with the vesicles to the Golgi matrix. Protoplasma 234, 51-64.

Langhans, M., Hawes, C., Hillmer, S., Hummel, E., and Robinson, D. G. (2007). Golgi regeneration after Brefeldin A treatment in BY-2 cells entails stack enlargement and cisternal growth followed by division. Plant Physiol. 145, 527-538.

Langhans, M., Meckel, T., Kress, A., Lerich, A., and Robinson, D. G. (2012). ERES (ER exit sites) and the "Secretory Unit Concept." J. Microsc. doi: 10.1111/j.1365-2818.2011.03597.x
Latijnhouwers, M., Gillespie, T., Boevink, P., Kriechbaumer, V., Hawes, C., and Carvalho, C. M. (2007). Localization and domain characterization of Arabidopsis golgin candidates. J. Exp. Bot. 58, 4373-4386.

Maizel, A., von Wangenheim, D., Federici, F., Haseloff, J., and Stelzer, E. H. K. (2011). High resolution live imaging of plant growth in near physiological bright conditions using light sheet microscopy. Plant J. 68, 377-385.

Matsuura-Tokita, K., Takeuchi, M., Ichihara, A., Mikuriya, K., and Nakano, A. (2006). Live imaging of yeast Golgi cisternal maturation. Nature 441, 939-940.

Osterrieder, A., Hummel, E., Carvalho, S. M., and Hawes, C. (2010). Golgi membrane dynamics after induction of a dominant-negative mutant Sarl GTPase in tobacco. J. Exp. Bot. 61, 405-422.

Paul, M. J., and Frigerio, L. (2007). Coated vesicles in plant cells. Semin. Cell Dev. Biol. 18, 471-478.

Renna, L., Hanton, S. L., Stefano, G., Bortolotti, L., Misra, V., and Brandizzi, F. (2005). Identification and characterization of At CASP, a plant transmembrane Golgi matrix protein. Plant Mol. Biol. 58, 109-122.

Richter, S., Geldner, N., Schrader, J., Wolters, H., Stierhof, Y.-D., Rios, G., Koncz, C., Robinson, D. G., and Jürgens, G. (2007). Functional diversification of closely related ARF-GEFs in protein secretion and recycling. Nature 448, 488-493.

Robinson, D. G., Herranz, M.-C., Bubeck, J., Pepperkok, R., and Ritzenthaler, C. (2007). Membrane dynamics in the early secretory pathway. CRC Crit. Rev. Plant Sci. 26, 199-225.

Robinson, D. G., Langhans, M., Saint-Jore-Dupas, C., and Hawes, C. (2008). BFA effects are tissue and not just plant specific. Trends Plant Sci. 13, 405-408.

Runions, J., Brach, T., Kühner, T., and Hawes, C. (2006). Photoactivation of GFP reveals protein dynamics within the endoplasmic reticulum membrane. J. Exp. Bot. 57, 43-50.

Saint-Jore, C. M., Evins, J., Batoko, H., Brandizzi, F., Moore, I., and Hawes, C. R. (2002). Redistribution of membrane proteins between the Golgi apparatus and endoplasmic reticulum in plants is reversible and not dependent on cytoskeletal networks. Plant J. 29, 661-678.

Schoberer, J., Runions, J., Steinkellner, H., Strasser, R., Hawes, C., and Osterrieder, A. (2010). Sequential depletion and acquisition of proteins during Golgi stack disassembly and reformation. Traffic 11, 1429-1444.

Sparkes, I., Runions, J., Hawes, C., and Griffing, L. (2009a). Movement and remodeling of the endoplasmic reticulum in nondividing cells of tobacco leaves. Plant Cell 21, 3937-3949.

Sparkes, I. A., Ketelaar, T., de Ruijter, N. C.A., and Hawes, C. (2009b). Grab a Golgi: laser trapping of Golgi bodies reveals in vivo interaction with the endoplasmic reticulum. Traffic 10, 567-571.
Sparkes, I., Tolley, N., Aller, I., Svozil, J., Osterrieder, A., Botchway, S., Mueller, C., Frigerio, L., and Hawes, C. (2010). Five plant reticulon isoforms share ER localisation, topology, ER membrane shaping properties and the ability to form both homo- and heterotypic interactions. Plant Cell 22, 1333-1343.

Stefano, G., Renna, L., Chatre, L., Hanton, S., Moreau, P., Hawes, C., and Brandizzi, F. (2006). In tobacco leaf epidermal cells the integrity of protein export from the endoplasmic reticulum and of ER export sites depends on active COPI machinery. Plant J. 46, 95-110.

Stefano, G., Renna, L., Moss, T., McNew, J. A., and Brandizzi, F. (2011). In Arabidopsis, the spatial and dynamic organization of the endoplasmic reticulum and Golgi apparatus is influenced by the integrity of the C-terminal domain of RHD3, anon-essential GTPase. Plant J. 69, 957-966.

Strating, J. R. P. M., and Martens, J. M. (2009). The p24 family and selective transport processes at the ER-Golgi interface. Biol. Cell 101, 495-509.

Tang, L. T., Wang, Y., Ong, Y. S., and Hong, W. (2005). COPII and exit from the endoplasmic reticulum. Biochim. Biophys. Acta 1744, 293-303.

Tolley, N., Sparkes, I., Eastmond, P. J., Hawes, C., and Frigerio, L. (2010). Transmembrane domain length is responsible for the ability of a plant reticulon to shape ER tubules in vivo. Plant J. 64, 411-418.

Wang, P., Hummel, E., Osterrieder, A., Meyer, A. J., Frigerio, L., and Hawes, C. (2011). KMS1 and KMS2, novel plant endoplasmic reticulum proteins involved in the early secretory pathway. Plant J. 66, 613-628.

Yang, Y. D., Elamawi, R., Bubeck, J., Pepperkok, R., Ritzenthaler, C., and Robinson, D. G. (2005). Dynamics of COPII vesicles and the Golgi apparatus in cultured Nicotiana tabacum BY-2 cells provides evidence for transient association of Golgi stacks with endoplasmic reticulum exit sites. Plant Cell 17, 1513-1531.

Zhang, C., Kotchoni, S. O., Samuels, A. L., and Szymanski, D. B. (2010). SPIKE1 signals originate from and assemble specialized domains of the endoplasmic reticulum. Curr. Biol. 20, 2144-2149.

Received: 26 October 2011; accepted: 29 March 2012; published online: 18 April 2012.

Citation: Hawes C (2012) The ER/Golgi interface-is there anything in-between? Front. Plant Sci. 3:73. doi: 10.3389/ fpls.2012.00073

This article was submitted to Frontiers in Plant Cell Biology, a specialty of Frontiers in Plant Science.

Copyright (c) 2012 Hawes. This is an open-access article distributed under the terms of the Creative Commons Attribution Non Commercial License, which permits non-commercial use, distribution, and reproduction in other forums, provided the original authors and source are credited. 